\title{
Allen, Danielle (2020): Politische Gleichheit. Frankfurter Adorno-Vorlesungen 2017
}

\author{
Berlin: Suhrkamp. 240 Seiten. $28 €$
}

\author{
Bernd Ladwig
}

Angenommen: 29. Juni 2021 / Online publiziert: 19. Juli 2021

(C) Der/die Autor(en) 2021

Die philosophische Diskussion über Gerechtigkeit wurde lange Zeit von Grundfragen der verteilenden Gerechtigkeit dominiert. Dieses sogenannte Distributionsparadigma sieht sich heute mit zwei Einwänden konfrontiert. Dem ersten Einwand zufolge ist der Fokus auf Verteilungsmuster zu statisch. Maßgeblich seien vielmehr die Beziehungen, die den Verteilungen zugrunde lägen und durch diese wiederum auch fortgeschrieben oder verändert würden. Der zweite Einwand lautet, dass das Distributionsparadigma nicht politisch genug sei. Es unterschätze die Bedeutung der Mitsprache über die Regelungen, aus denen dann erst bestimmte Verteilungsergebnisse hervorgingen.

In ihrem Buch „Politische Gleichheit“ kombiniert die in Harvard lehrende Politikwissenschaftlerin Danielle Allen beide Einwände. Sie möchte eine Theorie der Gerechtigkeit entwickeln, deren Zentrum egalitäre Beziehungen unter Bürgerinnen und Bürgern bilden. Diese Beziehungen sollten auf den Grundwert menschlicher Autonomie zugeschnitten sein. Autonomie ist für Allen ein wesentlicher Aspekt menschlichen Wohlergehens, und zu ihrer unverkürzten Verwirklichung gehörten öffentliche genauso wie private Freiheiten. Zwar habe schon Rawls mit seinem ersten Gerechtigkeitsgrundsatz den Anspruch erhoben, die (negativen) Freiheiten der Modernen und die (positiven) Freiheiten der Alten gleichgewichtig zur Geltung zu bringen. Aus seiner nichtidealen Theorie gehe indes hervor, dass er im Zweifelsfall die politische Dimension der Freiheit für die persönliche opfern würde.

Ein solches Opfer möchte Allen unbedingt ausschließen. Für sie ist die Demokratie der einzige Weg, der zu Gerechtigkeit führt. Für unsere Autonomie sei sie sowohl instrumentell als auch intrinsisch unverzichtbar. Wahres Wohlergehen sei ohne politische Partizipation nicht zu erlangen. Deshalb wendet sich Allen auch ge-

Bernd Ladwig $(\bowtie)$

Institut für Philosophie, Freie Universität Berlin, Berlin, Deutschland

E-Mail: bernd.ladwig@fu-berlin.de 
gen Vorschläge, mehr Menschen für eine verteilungspolitisch vorteilhafte Migration zu gewinnen, indem man den einwandernden Menschen politische Mitsprachemöglichkeiten vorenthält. Wer einwandern dürfe, müsse auch Aussicht auf politische Mitgliedschaft haben oder zumindest mittelst zivilgesellschaftlicher Organisationen politisch mitreden dürfen.

Politische Gleichheit liegt nach Allen vor, wenn fünf Bedingungen erfüllt seien. Erstens müssten alle politischen und auch sozialen Beziehungen frei sein von willkürlicher Beherrschung (,domination“). Zweitens müssten alle die gleichen Chancen auf Zugang zu den politischen Entscheidungszentren haben. Drittens müssten politische Entscheidungen auf der Grundlage eines epistemischen Egalitarismus zustande kommen, worunter Allen die richtige Verbindung von Expertenwissen und dem Wissen gewöhnlicher Menschen versteht. Viertens müssten die Bürgerinnen und Bürger ein Ethos der Gegenseitigkeit kultivieren: Indem sie füreinander ansprechbar bleiben, erkennen sie wechselseitig ihre Beiträge für das Gemeinwesen an, leisten Wiedergutmachung für Missstände und sorgen so für ein ausgewogenes Verhältnis der Handlungsmacht, die unter ihnen herrscht. Fünftens schließlich sollten sie sich als Miteigentümerinnen an den politischen Institutionen verstehen. Auch wenn Allen hier vage bleibt, scheinen doch die Nichtbeherrschung und die Gegenseitigkeit zusammen den Kern der politischen Gleichheit zu ergeben. Damit kommt der relationale Charakter ihres Gerechtigkeitsverständnisses zur Geltung: Es geht ihr um Beziehungen, in denen Bürgerinnen und Bürger in einer von Willkür freien Weise aufeinander Bezug nehmen, um gemeinsam ein System gleicher persönlicher und politischer Freiheiten zu gestalten.

Das politische Gleichheitsverständnis bildet den Leitgesichtspunkt, unter dem dann auch Fragen des Sozialen und der Wirtschaft für die Gerechtigkeit relevant werden. Politische Gleichheit verweist erstens auf das Ideal einer sozialen Welt der Differenz ohne Herrschaft. Während Differenzen unvermeidlich seien, wo immer Menschen von ihren bürgerlichen Freiheiten Gebrauch machten, sollten sich Herrschaftseffekte, wie sie etwa als Nebenfolge sozialer Segregation drohten, vermeiden lassen. Auf wirtschaftlichem Gebiet verlange politische Gleichheit nach ökonomischer Ermächtigung. Allen versteht darunter einen demokratisch kontrollierten Stakeholder-Kapitalismus sowie Mitentscheidungsmöglichkeiten der Beschäftigten nach dem Vorbild des deutschen Mitbestimmungsgesetzes. Die Produktionsverhältnisse seien dabei für die Gerechtigkeit wichtiger als Fragen der (Um-)Verteilung.

Vor allem aber hält es Allen für einen Fehler, Fragen der Wirtschaftsorganisation von sozialen Fragen etwa der Gestaltung von Bildungs-, Verkehrs- und Wohnverhältnissen zu trennen. Sie möchte das Ökonomische und das Soziale vielmehr unter dem gedanklichen Dach einer vernetzten Gesellschaft miteinander verbinden. Die vernetzte Gesellschaft ist Allens Antwort auf das Problem, das der Gebrauch der persönlichen Freiheiten unvermeidlich Unterschiede erzeugt, die die politische Gleichheit zu untergraben drohen. Freie Menschen werden etwa ihr Recht wahrnehmen, mit Menschen zusammenzuleben, die ihnen ähnlich sind, auch wenn dies sozial homogene Stadtviertel zur Folge hat. Umso wichtiger wären Anreize zum sozialen Brückenbau. Zu denken wäre etwa an gut ausgestattete staatliche Schulen, die von Kindern unterschiedlicher sozialer und ethnischer Herkunft besucht werden, oder an eine gegen Gentrifizierung gerichtete Wohnungspolitik. 
Wer Allens Buch als eine Alternative zur Gerechtigkeitstheorie à la Rawls liest, wird es eher enttäuscht aus der Hand legen. Dazu fehlt es ihm an begrifflicher Klarheit, argumentativer Stringenz und Tiefe der Begründung. Allen ignoriert zum Beispiel den Einwand, dass eine autonomiebezogene Begründung von Demokratie daran scheitere, dass wir als Teil eines nach Mehrheitsregeln regierten Demos eben nicht unbedingt auch individuell selbstbestimmt blieben.

Vor allem aber geht Allens Kritik an Rawls daran vorbei, dass dieser fast durchweg auf einer höheren Abstraktionsstufe verbleibt. Rawls sagt wenig darüber, welche institutionellen Regelungen und kulturellen Einstellungen erforderlich sind, damit alle die gleichen Grundfreiheiten genießen können. Seine Gerechtigkeitsgrundsätze bilden allgemein formulierte Bezugspunkte für Fragen der Umsetzung, die mit jedem Anwendungsschritt tiefer in die Besonderheiten geschichtlich geprägter Umstände hineinführen.

Auch Allen bleibt vergleichsweise allgemein, aber ihrem methodischen Vorbild Aristoteles folgend legt sie ihr Augenmerk nicht zuletzt auf die sozialstrukturellen, kulturellen und demografischen Bedingungen für bürgerschaftliche Gleichheit. Darin liegt die eigentliche Stärke ihres Buches: Sie macht deutlich, dass zur Verwirklichung gleicher Autonomie für alle mehr gehört als grundrechtliche Garantien und Instrumente der Umverteilung. Unsere formal gleichen Freiheiten bedürften einer Infrastruktur aus Voraussetzungen, die wir nur durch Zusammenführung unseres gemeinsamen geistes- und sozialwissenschaftlichen Sachverstandes unverkürzt erfassen könnten.

Funding Open Access funding enabled and organized by Projekt DEAL.

Open Access Dieser Artikel wird unter der Creative Commons Namensnennung 4.0 International Lizenz veröffentlicht, welche die Nutzung, Vervielfältigung, Bearbeitung, Verbreitung und Wiedergabe in jeglichem Medium und Format erlaubt, sofern Sie den/die ursprünglichen Autor(en) und die Quelle ordnungsgemäß nennen, einen Link zur Creative Commons Lizenz beifügen und angeben, ob Änderungen vorgenommen wurden.

Die in diesem Artikel enthaltenen Bilder und sonstiges Drittmaterial unterliegen ebenfalls der genannten Creative Commons Lizenz, sofern sich aus der Abbildungslegende nichts anderes ergibt. Sofern das betreffende Material nicht unter der genannten Creative Commons Lizenz steht und die betreffende Handlung nicht nach gesetzlichen Vorschriften erlaubt ist, ist für die oben aufgeführten Weiterverwendungen des Materials die Einwilligung des jeweiligen Rechteinhabers einzuholen.

Weitere Details zur Lizenz entnehmen Sie bitte der Lizenzinformation auf http://creativecommons.org/ licenses/by/4.0/deed.de. 